\title{
Auswertung der von Kautsky, Gaubatz und Greiff gemessenen Adsorptionsisothermen von Gasen an Siloxen und Bromsiloxenen. I
}

\author{
Von Ewald Dadbach
}

\author{
Aus dem Institut für Siliciumchemie der Universität Marburg \\ (Z. Naturforschg. 8a, 186-190 [1953]; eingegangen am 7. August 1952)
}

\begin{abstract}
Messungen der Adsorption von $\mathrm{CH}_{4}, \mathrm{C}_{2} \mathrm{H}_{6}, \mathrm{C}_{3} \mathrm{H}_{8}$ und $\mathrm{CO}_{2}$ an Siloxen und Bromsiloxenen ${ }^{1.2}$ werden mit Hilfe der Langmuirschen Methode ausgewertet. Nur die Isothermen des $\mathrm{CH}_{4}$ und des $\mathrm{CO}_{2}$ lassen sich im ganzen untersuchten Druckbereich durch eine einzige Langmuir-Gleichung interpretieren. Demgegenüber sind zur Darstellung von 3 der 4 Isothermen des Äthans und aller Isothermen des Propans 2 verschiedene Langmuir-Gleichungen nötig, von denen eine bei niederen und die andere bei höheren Drucken gültig ist. Diese Erscheinung wird mit den in den adsorbierten Molekeln induzierten Dipolen und den Kraftwirkungen zwischen diesen in Verbindung gebracht.
\end{abstract}

$\mathrm{K}$ autsky und Mitarb. ${ }^{1,2}$ haben Siloxen und einige seiner Derivate zu Adsorptionsmessungen herangezogen. Diese Stoffe vermögen zu unseren Kenntnissen über die Adsorption auf Grund ihrer besonderen Eigenschaften interessante Beiträge zu liefern. Siloxen ist ein fester Stoff der Zusammensetzung $\left(\mathrm{Si}_{6} \mathrm{O}_{3} \mathrm{H}_{6}\right)_{\mathrm{n}}$ und besitzt infolge seiner permutoiden Netzstruktur ${ }^{3,4}$ eine in bezug auf chemische Konstitution und auf Größe definierte Grenzfläche. Letztere läßt sich durch chemische Reaktionen (Substitutionen der H-Atome) in ihrer $\mathrm{Zu}$ sammensetzung verändern, so daß bei den Derivaten des Siloxens der Einfluß einer definierten chemischen Variation der Grenzfläche auf die Adsorption ermittelt werden kann, unter Erhalt der Größe der Grenzfläche und ihres permutoiden Charakters.

Kautsky und Gaubatz ${ }^{1}$ haben die Adsorption von $\mathrm{CO}_{2}$ und $\mathrm{C}_{2} \mathrm{H}_{6}$ an Siloxen, Mono-, Tri- und Penta-Bromsiloxen und Kautsky und Greiff ${ }^{2}$ die Adsorption von $\mathrm{CH}_{4}$ und $\mathrm{C}_{2} \mathrm{H}_{6}$ an Siloxen, Mono- und Tribromsiloxen und von $\mathrm{C}_{3} \mathrm{H}_{8}$ an Siloxen und Monobromsiloxen, alles bei Drucken unterhalb 1 at und bei $20^{\circ} \mathrm{C}$ gemessen ${ }^{5}$.

Kautsky und Greiff ${ }^{2}$ ermittelten aus der gra-

${ }^{1}$ H. Kautsky u. E. Gaubatz, Z. anorg. allg. Chem. 191, 382 [1930].

${ }^{2}$ H. Kautsky u. F. Greiff, Z. anorg. allg. Chem. 236, 124 [1938].

${ }^{3}$ H. Kautsky u. G. Herzberg, Z. anorg. allg. Chem. 147, 81 [1925].

4 Definition der permutoiden Netzstruktur: Verhältnis von Zahl der Atome in der Oberfläche zu Gesamtzahl der Atome in der festen Phase $=1$.

5 Nach den Angaben der Autoren ist begreiflicherweise den Messungen von Kautsky u. Greiff ${ }^{2}$, eini- phischen Darstellung der Adsorptionsisothermen nach Langmuir ${ }^{6}$ nur die Sättigungswerte $A_{\mathrm{s}}$ (zur Bezeichnung s. später) für $\mathrm{CH}_{4}, \mathrm{C}_{2} \mathrm{H}_{6}$ und $\mathrm{C}_{3} \mathrm{H}_{8}$ jeweils an Siloxen.

Die vorliegende Arbeit befaßt sich mit einer vollständigen graphischen und rechnerischen Auswertung der Meßergebnisse von Kautsky und Mitarb. nach der Methode von Langmuir ${ }^{6}$ und vergleicht die Ergebnisse mit den nach der Methode von Brunauer, Emmett und Teller ${ }^{7}$ zu erhaltenden. In einer anschließenden Diskussion wird versucht, den beobachteten Isothermenverlauf von den Kraftwirkungen her zu verstehen, die von den adsorbierenden und den adsorbierten Teilchen ausgehen.

Beschreibung des Auswertungsverfahrens

Die Langmuir-Gleichung wird in folgender Form angewandt:

$$
A=\left(A_{\mathrm{s}} \cdot \mathrm{P}\right) /(K+P),
$$

worin $A=$ adsorbierte Menge in Molen Gas pro Mol Adsorbens ${ }^{8}, \quad A_{\mathrm{s}}=$ maximal adsorbierbare Menge in Molen Gas pro Mol Adsorbens bei $20^{\circ} \mathrm{C}$, $P=$ Gasdruck in Torr und $K=$ Konstante.

gen Verbesserungen gegenüber der grundlegenden Arbeit von Kautsky und Gaubatz ${ }^{1}$ zufolge, die größere Genauigkeit zuzuerkennen.

6 I. Langmuir, J. Amer. chem. Soc. 38, 2221 [1916]; 40, 1369 [1918].

7 S. Brunauer, P. Emmett u. E. Teller, J. Amer. chem. Soc. 60, 309 [1938].

${ }^{8} \mathrm{Als}$ Mol Adsorbens ist stets das Gewicht in $\mathrm{g}$ der Einheit $\mathrm{Si}_{6} \mathrm{O}_{3} \mathrm{H}_{n} \mathrm{Br}_{6-\mathrm{n}}(\mathrm{n}=6,5,3$ oder 1$) \mathrm{zu}$ verstehen; z. B. ist ein Mol Siloxen $(\mathrm{n}=6) 222 \mathrm{~g}$. 
Gl. (1) ist bei allen Isothermen der Arbeiten von Kautsky und Mitarb. ${ }^{1,2}$ bereits bei den niedrigsten Meßdrucken gültig. Dies ist eine Sonderheit des Siloxens gegenüber vielen anderen Adsorbentien.

\section{Ergebnisse der Auswertung}

Die nach Gl. (1) graphisch aus dem Diagramm $P / A$ gegen $P$ erhaltenen Zahlen von $A_{\mathrm{s}}$ und $K$ sind in Tab. 1 zusammengestellt. Für den Fall, daß in den Arbeiten ${ }^{1}$ und ${ }^{2}$ gleiches Adsorbens und gleicher Sorbens verwandt wurden, sind in Tab. 1 nur die aus ${ }^{2}$ gewonnenen Werte aufgenommen, da die aus ${ }^{1}$ ermittelten Zahlenangaben nur wenig hiervon abweichen außer bei einem, in Tab. 1 in Anm. $\dagger$ besonders angegebenen Fall.

Eine Reihe von Isothermen (花han an Mono-, Tri- und Pentabromsiloxen, Propan an Siloxen und Monobromsiloxen) müssen durch zwei Langmuir- sche Gleichungen dargestellt werden, von denen eine im Anfangsteil I der betr. Isotherme (bei niederen Drucken) und die andere im Endteil II (bei relativ höheren Drucken) gilt ${ }^{9}$. Utber die Grenzen der Bereiche I und II unterrichtet Tab. 1.

Die Isothermen mit den Gebieten I und II sollen durch die Abb. 1-2 verdeutlicht werden. Man erkennt bei jeder Isothermen deutlich die beiden Bereiche, in die sich die Meßpunkte unterteilen lassen.

\section{Charakter der Adsorption}

Mit Hilfe der nur auf Substanzen unterhalb ihrer kritischen Temperatur anwendbaren Methode von Brunauer, Emmett und Teller ${ }^{7}$ erhält man für $\mathrm{C}_{2} \mathrm{H}_{6}, \mathrm{C}_{3} \mathrm{H}_{8}$ und $\mathrm{CO}_{2}$ Werte von $A_{\mathrm{s}}$ und $K$, die mit denen der Tab. 1 befriedigend übereinstimmen. Aus

${ }^{9} \mathrm{D}$. h. im Diagramm $P / A$ gegen $P$ treten zwei Geraden auf, desgl. im Diagramm $1 / A$ gegen $1 / P$.

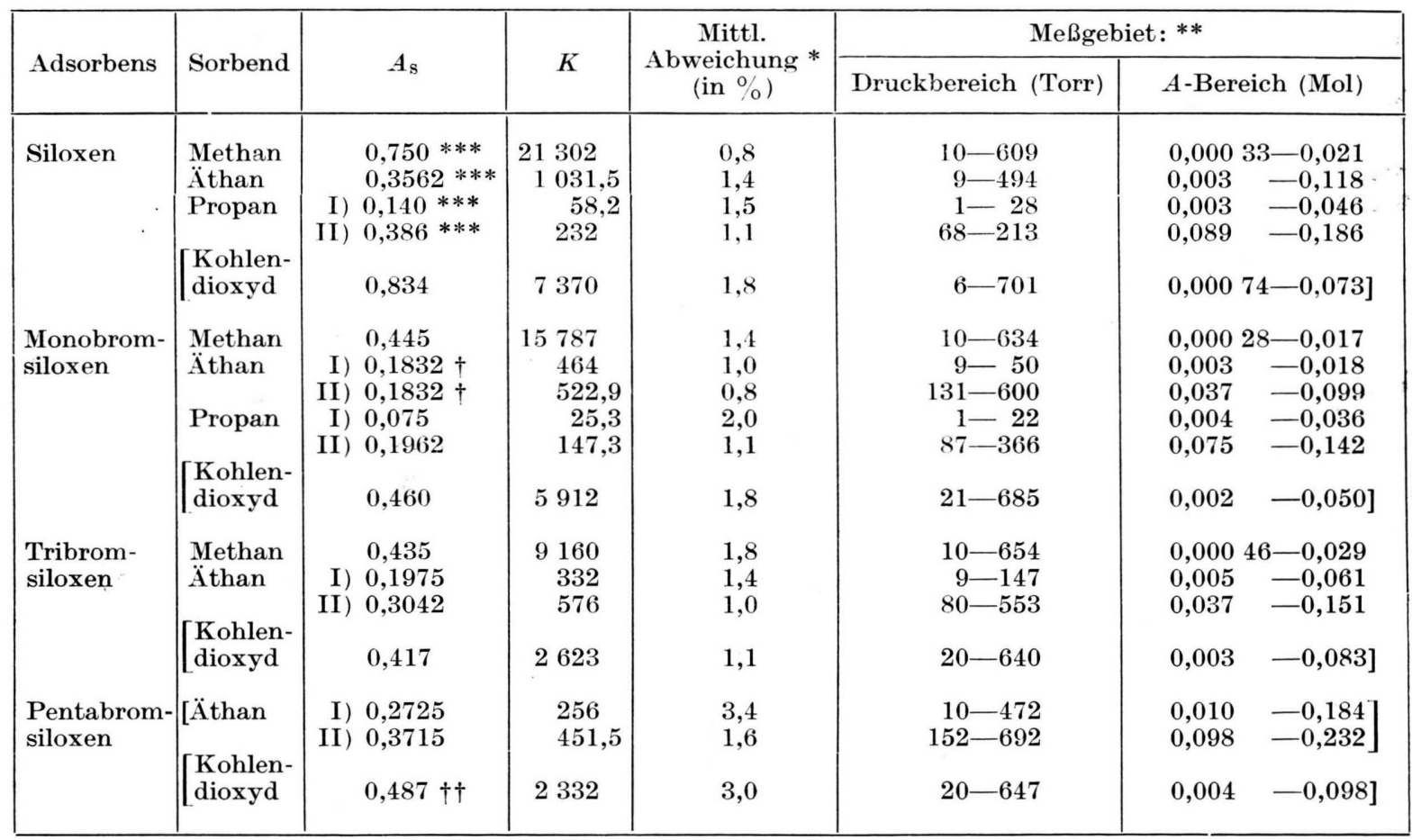

Tab. 1. Die Angaben in [] sind aus der Arbeit von Kautsky u. Gaubatz ${ }^{1}$ errechnet, alle anderen aus der Arbeit von Kautsky u. Greiff ${ }^{2}$.

* Mittl. Abweichung zwischen den nach Gl. (1) aus den $A_{\mathrm{s}}$ - und $K$-Werten errechneten Isothermen und den gemessenen $A$-Werten.

** Im Druckbereich abgerundet auf Torr, im $A$-Bereich auf 3 Stellen hinter dem Komma (außer bei 4 Werten).

*** Diese Werte sind bereits bei Kautsky u. Greiff ${ }^{2}$ angegeben.

† Die Auswertung der Messungen von Kautsky u. Gaubatz ${ }^{1}$ ergiht in diesem Fall abweichende $\boldsymbol{A}_{\mathrm{s}}-\mathrm{und} \boldsymbol{K}$-Werte:

Bereich I (20-349 Torr bzw. 0,007-0,067 Mol) : $A_{\mathrm{s}}=0,1324 ; K=352$,

Bereich II (276-548 Torr bzw. 0.058-0,092 Mol): $A_{\mathrm{s}}=0,2222 ; K=783,5$.

++ Die 3 letzten Meßpunkte (Bereich $348-647$ Torr bzw. 0,057 bis 0,098 Mol) lassen sich mit einem mittl Fehler von $0,3 \%$ durch die Werte $A_{s}=0,5868$ und $\boldsymbol{K}=3213$ interpretieren. Ohne diese 3 Meßpunkte wird der mittl Fehler in Tab. 1 statt 3 nur $1,2 \%$. - Vermutlich sind für diese Isothermenaufspaltung Meßfehler verantwortlich zu machen, da beim $\mathrm{CO}_{2}$ sonst nirgendwo 2 Bereiche auftreten. 
$K$ läßt sich nach der BET-Theorie ${ }^{7}$ die ungefähre Größe der mittleren Adsorptionswärme errechnen. An allen vier Adsorbenzien ergeben sich für $\mathrm{C}_{2} \mathrm{H}_{6}$ Werte von 3-6 kcal/Mol, für $\mathrm{C}_{3} \mathrm{H}_{8} 5-7$, und für $\mathrm{CO}_{2} 2-4$. Also muß physikalische (ungehemmte) Adsorption vorliegen. Letzteres dürfte erst recht für Methan zutreffen. Aus der Gültigkeit der Lang-

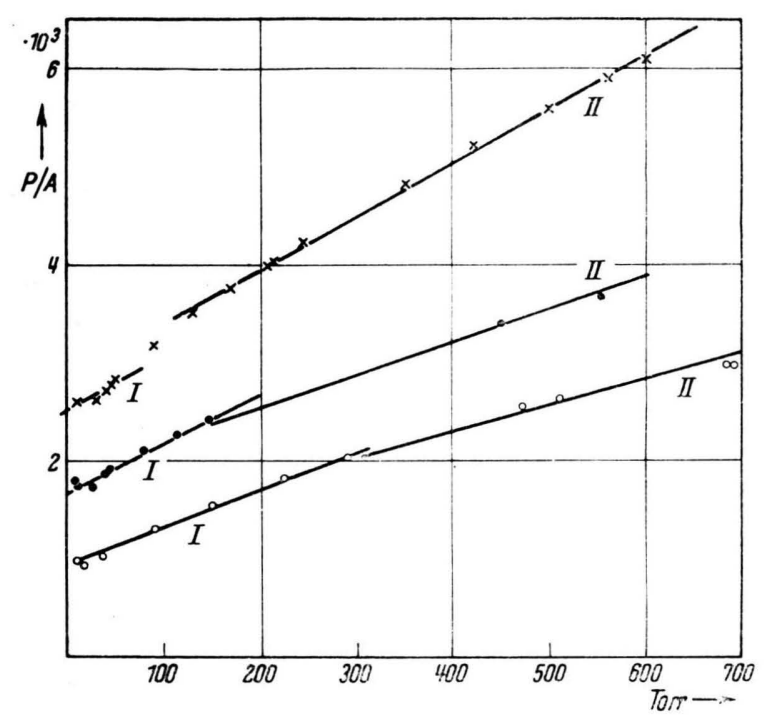

Abb. 1. $P / A$ - $P$-Diagramm] für Äthan an Bromsiloxenen.

Meßpunkte: $\times$ Monobromsiloxen, - Tribromsiloxen, o Pentabromsiloxen.

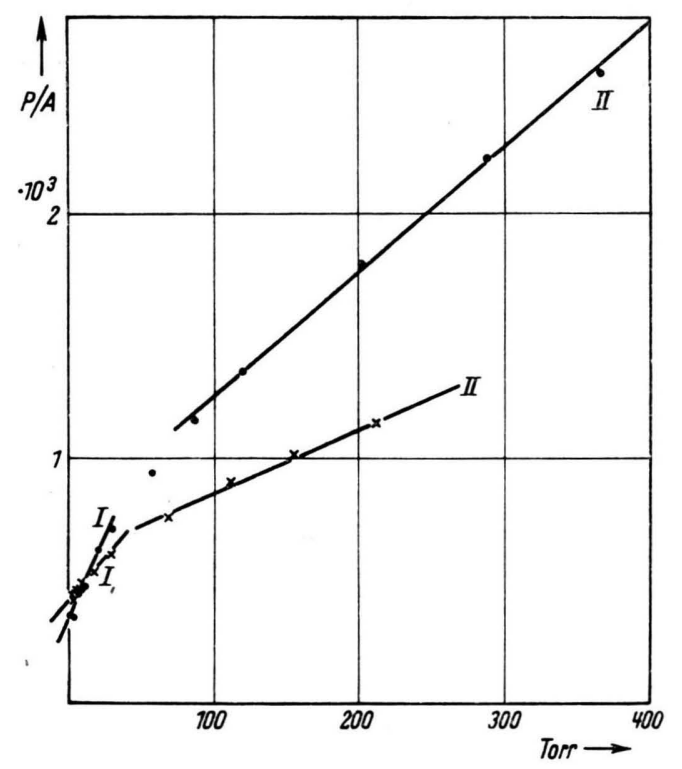

Abb. 2. $P / A$ - $P$-Diagramm für Propan. MeBpunkte: $\times$ Siloxen, - Monobromsiloxen. muirschen Gleichung folgt ferner, daß die Adsorption im untersuchten Druckbereich nur zur Ausbildung monomolekularer Schichten führt.

Der Verlauf der $K$-Werte bei successiver Substitution der H-Atome der Siloxenoberfläche durch Br (Abb. 3)

Die $K$-Werte eines Sorbenden nehmen beim Übergang von Siloxen zu Pentabromsiloxen durchweg ab, wobei man bei Äthan an den Bromsiloxenen und bei Propan an allen Adsorbenzien die beiden Isothermenteile I und II getrennt betrachten muß.

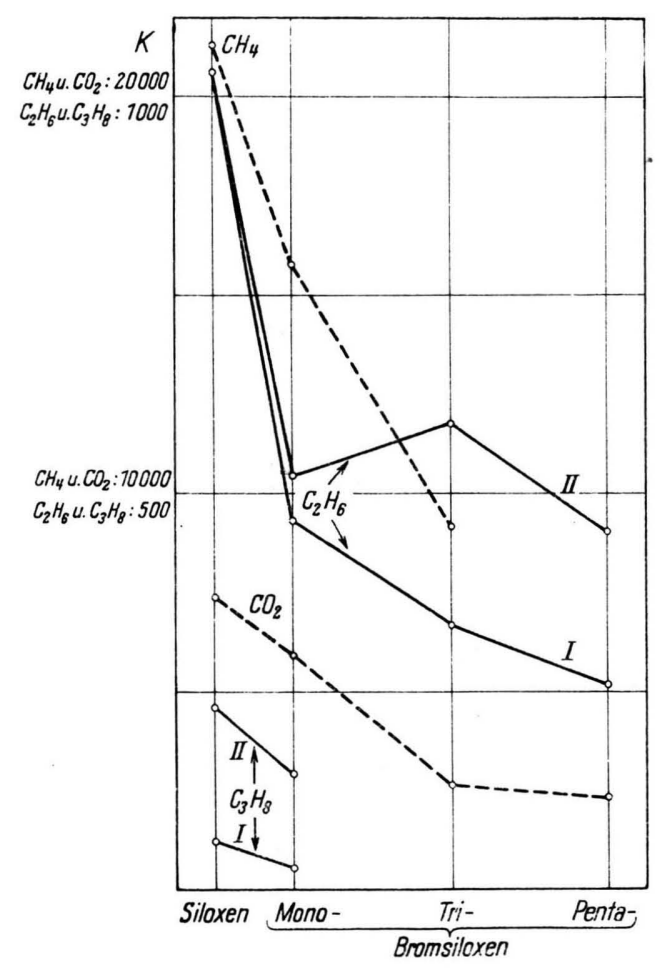

Abb. 3. $K$-Werte.

In 1. Näherung kann man aus diesem Gang auf eine Zunahme der Kraftwirkungen zwischen Oberfläche und adsorbierten Molekeln beim Übergang vom Siloxen zum Pentabromsiloxen schließen. Das ist plausibel, da die leichten H-Atome des Siloxens fortschreitend durch schwere Br-Atome ersetzt werden, die beim Pentabromsiloxen weitgehend (wenn nicht völlig) den Charakter der Oberfläche bestimmen.

Beim Übergang vom Isothermenteil I nach II nimmt $K$ stets zu. Dies würde, wiederum in 1. Näherung, bedeuten, daß im Bereich I stärkere Wechsel- 
wirkungen zwischen Oberfläche und adsorbierten Molekeln herrschen, als im Bereich II. Der größere Wert von $K$ im Gebiet II wird von später zu besprechenden Gesichtspunkten aus verständlich erscheinen.

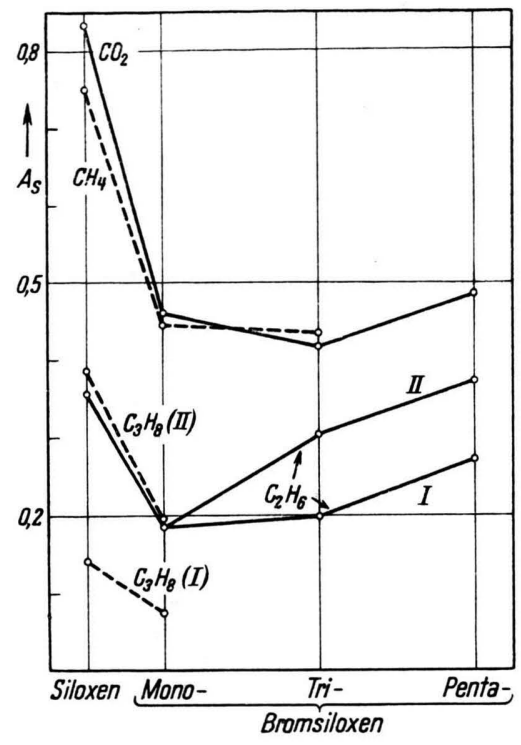

Abb. 4. $A_{\mathrm{s}}-$ Werte.

Der Verlauf der $A_{\mathrm{s}}$-Werte bei successiver Substitution der H-Atome der Siloxenoberfläche durch Br (Abb.4)

Der $A_{\mathrm{s}}$-Wert eines Gases nimmt beim Übergang von Siloxen zu Monobromsiloxen stark ab, um dann bei weiterem Fortschreiten zu Tri- und Pentabromsiloxen durchweg wieder langsam zuzunehmen (wobei, wie zuvor, die Bereiche I und II getrennt $\mathrm{zu}$ betrachten sind). Im Monobromsiloxen bewirken die Br-Atome offensichtlich eine Verringerung der für die Adsorption wirksamen Oberfläche im Vergleich zum Siloxen ${ }^{10}$; im Pentabromsiloxen hingegen haben sie die Funktion der $\mathrm{H}$-Atome des

${ }^{10}$ Diese Feststellung findet sich bereits bei K a u ts k y und Greiff ${ }^{2}$.

11 Ausgenommen $\mathrm{C}_{2} \mathrm{H}_{6}$ an Monobromsiloxen, wo beide $A_{\mathrm{s}}$-Werte gleich sind. S. jedoch Anm. $\uparrow$ zu Tab. 1.

${ }^{12}$ Beim $\mathrm{CO}_{2}$ an Pentabromsiloxen können die Meßpunkte bei höherem Druck zwar durch eine zweite Langmuir-Gleichung dargestellt werden - vgl. Anm. $\dagger \dagger$ zu Tab. 1; jedoch dürfte dies durch Meßfehler zu erklären sein - vgl. Anm. 5.

${ }^{13}$ S. z. B. S. Brunauer, The Adsorption of Vapors and Gases, Princeton 1945.

14 Auszuschließen ist ihr Zustandekommen durch eine zu langsame Gleichgewichtseinstellung bei der
Siloxens hinsichtlich der maximal adsorbierbaren Menge weitgehend übernommen.

Die $A_{\mathrm{s}}$-Werte des Gebietes II eines Gases liegen durchweg höher ${ }^{11}$ als die des Gebietes I. Eine Erklärung hierfür wird weiter unten versucht.

Die Isothermenteile I und II und Begründungen für ihr Auftreten

Eine einzige Langmuir-Gleichung vermag die Isothermen des $\mathrm{CH}_{4}$ und des $\mathrm{CO}_{2}$ an allen Adsorbenzien ${ }^{12}$ und des $\mathrm{C}_{2} \mathrm{H}_{6}$ an Siloxen darzustellen.

Alle anderen Isothermen (vgl. Tab. 1 u. Abb. 1 u. 2) müssen in die beiden Gebiete I und II aufgeteilt werden. Von diesen Isothermen wird im folgenden Teil dieses Kapitels allein die Rede sein.

Die Isothermenteile I und II konnten bei einer großen Zahl von Rechnungen sowohl unter Zuhilfenahme der BET-Theorie ${ }^{7}$ als auch der Polanyischen Potentialtheorie ${ }^{13}$ nicht zum Versch winden gebracht (d. h. zu einer einzigen Kurve verschmolzen) werden. Dies spricht dafür, daß die beiden Gebiete eine physikalische Bedeutung besitzen ${ }^{14}$.

1. Es ist kaum möglich, das Auftreten der beiden Gebiete damit zu erklären, daß es in der Oberfläche des Adsorbens zwei Sorten von Aktivstellen gebe ${ }^{15}$, weil sich die beobachteten Isothermen nicht durch eine Gleichung der Art

$$
A=\frac{A_{\mathrm{s}}^{\prime} P}{K^{\prime}+P}+\frac{A_{\mathrm{s}}^{\prime \prime} P}{K^{\prime \prime}+P}
$$

darstellen lassen.

2. Deshalb ist es vertretbar, eine andere, wahrscheinlichere Erklärung für das Auftreten der Isothermenteile I und II anzuführen. Die polaren Si-H-, Si-O- und Si-Br-Gruppen der Adsorbenzien, welche verhältnismäßig starke Dipole der Größenordnung $10^{-18}[\mathrm{esE} \cdot \mathrm{cm}]$ darstellen ${ }^{16}$, vermögen in den absorbierten Molekeln ein Dipolmoment zu erzeugen. Die Komponente dieses Moments senkrecht zur Siloxenoberfläche bewirkt bei größerer Entfer-

Adsorption, da von Kautsky und Gaubatz ${ }^{1}$ bei Druckerhöhung und Druckerniedrigung durchgeführte Messungen einer Adsorptionsisothermen zum gleichen $A$-Wert führten.

15 Auf diese Weise ist eine Reihe gleichartiger Knicke im $P / A$ - $P$-Diagramm bei der Adsorption von Chlor an $\mathrm{SiO}_{2}$ von Brunauer ${ }^{13}$ erklärt worden; jedoch erscheint seine Darstellung etwas unsicher.

16 Si-O-Bindung: $2,8 \cdot 10^{-18}$

Si-H-Bindung: $0,35 \cdot 10^{-18}$ Aus den ElektronegatiSi-Br-Bindung: $2,0 \cdot 10^{-18}$ vitäten nach der Paulingschen Formel abgeschätzt. (R. Sauer u. D. Mead, J. Amer. chem. Soc. 68, 1796 [1946]. 
nung der absorbierten Molekeln voneinander eine Dipol-Abstoßung zwischen diesen (Energie prop. $r^{-3}$ ), während bei geringerer Entfernung die Van der Waals-Anziehung (Energie prop. $r^{-6}$ ) überwiegt.

Zur Stützung dieser Ausführungen ist folgendes erwähnenswert: Unter Annahme einer gewellten Struktur des Sil xennetzes, tetraedischer Bindungsrichtungen des Siliciums und eines Winkels Si-O-Si von $180^{017}$ und unter Berücksichtigung der umgebenden Bindungen bis zu etwa $20 \AA$ Entfernung von dem absorbierten Teilchen läßt sich aus den Feldtstärken ${ }^{18}$ der als Dipole bzw. lineare Quadrupole betrachteten Si-H-, Si-Br- und Si-O-Si-Bindungen die in den adsorbierten Molekeln induzierte Komponente des Dipolmoments senkrecht zur Netzebene ${ }^{19}$ abschätzen. Daraus kann man für gewisse Lagen der adsorbierten Molekeln und hexagonale Packung derselben auf der Siloxenoberfläche die Abstoßungsenergie ${ }^{18}$ auf Grund dieser Komponente ausrechnen. Wenn man diese Energie mit der Van derWaals-Anziehung zwischen den adsorbierten Teilchen ${ }^{20}$ vergleicht, so erhält man Gleichheit der beiden Energien bei mittleren Entfernungen der Teilchen voneinander, welche zwischen 10 und $100 \AA$ liegen. Errechnet man andererseits aus der molaren (zweiseitigen) Oberfläche des Siloxens $\left(6 \cdot 10^{25} \AA^{2}\right)^{21}$ und unter Annahme der gleichen hexagonalen Packung die mittlere Entfernung für die Übergangsgebiete I/II aus den $A$-Werten der Tab. 1, so erhält man wiederum Werte im gleichen Bereich, nämlich zwischen 30 und $80 \AA$.

Ferner ist erwähnenswert, daß sich 2 Langmuirsche Adsorptionsisothermen, welche dem Typ der durch Auswertung der Experimente erhaltenen Bereiche I und II gleichen, aus der Gleichung für isothermes Gleichgewicht zwischen gasförmiger und adsorbierter Phase ${ }^{13}$ ableiten lassen ${ }^{22}$. Dazu muß man auf die Gasphase die ideale Zustandsgleichung und auf die adsorbierte Phase die Van der Waals'sche Zustandsgleichung anwenden, letztere mit der Van

17 Zur räumlichen Struktur des Siloxens, vgl. z. B. Ewald Daubach, Diss. Marburg 1951, S. $197 \mathrm{ff.}$; ferner ${ }^{21}$.

${ }^{18}$ Formeln s. z. B. O. K. Rice, Electronic Structure and Chemical Binding, Mc. Graw Hill, New York 1940.

19 Größenordnung $10^{-20}$ [esE $\cdot \mathrm{cm}$ ].

${ }^{20}$ Formel z. B. nach Eucken, Lehrb. d. Chem. Phys. 3. Aufl. Bd. II, 218: $E=\left(3 \alpha^{2} I\right) /\left(4 r^{6}\right) . \quad(\alpha=$ Polarisierbarkeit, $I \approx$ erstes Ionisationspotential für adsorbierte Teilchen.)

${ }^{21}$ H. P. Siebel, Diss. Marburg 1952, S. $31 \mathrm{ff}$. der Waals-Konstanten $a_{\mathrm{I}}<0$ (für Gebiet I) wegen der Abstoßung zwischen den adsorbierten Teilchen in diesem Bereich ${ }^{23}$, und mit $a_{\mathrm{II}}>0$ (für Gebiet II) wegen der dort überwiegenden Anziehung 24. Die Größen $K$ und $A_{\mathrm{s}}$ der so erhaltenen 2 LangmuirIsothermen erfüllen die Beziehungen $K_{\mathrm{II}}>K_{\mathrm{I}}$ und $A_{\mathrm{s}, \mathrm{II}}>A_{\mathrm{s}, \mathrm{I}}$, was in Übereinstimmung mit denWerten der Tab. 1 steht.

Die Ausführungen der beiden letzten Abschnitte können nur vorläufiger Natur sein. Sie bedürfen noch wesentlicher Ergänzungen und möglicherweise neuer Gesichtspunkte. Die Folgerungen, die man aus ihnen ziehen kann, stehen jedoch in Übereinstimmung mit den Tatsachen.

Die Isothermen, die keine Aufteilung zeigen

Die Tatsache, daß bei den Adsorptionsisothermen des $\mathrm{CH}_{4}$, des $\mathrm{CO}_{2}$ und des $\mathrm{C}_{2} \mathrm{H}_{6}$ (für letzteres nur an Siloxen) keine zwei Gebiete auftreten, läßt sich wenigstens für Methan und Kohlendioxyd unter Zuhilfenahme der zuletzt gegebenen Erklärung für das Auftreten der beiden Bereiche gut verstehen. Die Adsorption von $\mathrm{CH}_{4}$ liegt bei so kleinen $A$ Werten, daß das Gebiet II im Meßbereich noch nicht erreicht wird, was auch in Übereinstimmung mit den oben erwähnten Abschätzungen über die Energien steht. Auch die $\mathrm{CO}_{2}$-Isothermen gehen nur bis zu mäßig großen $A$-Werten, so daß hier das gleiche gelten dürfte. Außerdem ist die $\mathrm{CO}_{2}$-Molekel zum Unterschied von allen anderen angewandten Adsorbenzien ein linearer Quadrupol, was ebenfalls von Bedeutung sein könnte. Für die Erklärung der Tatsache, daß bei der Adsorption des Äthans an Siloxen kein Gebiet II auftritt, sind ins einzelne gehende Betrachtungen und Rechnungen erforderlich, die noch durchgeführt werden sollen.

Den Herren Prof. E. Hückel, H. Kautsky u. F. H. M üller danke ich für wertvolle Diskussionen.

${ }^{22}$ Für die Ableitung einer Langmuir-Isothermen aus dem Gleichgewicht Gas/adsorbierte Phase vgl. Bruna uer ${ }^{13}$.

${ }^{23}$ Für eine Van der Waals-Gleichung für sich abstoßende Teilchen vgl. auch A. Magnus, Z. physik. Chem., Abt. A 142, 401 [1929].

24 Anders ausgedrückt: Es sind die beiden Gleichgewichte Gas/ads. Phase I und Gas/ads. Phase II betrachtet; für jedes Gleichgewicht wird eine LangmuirIsotherme abgeleitet. 